\title{
Computing and Controlling Basins of Attraction in Multistability Scenarios
}

\author{
John Alexander Taborda ${ }^{1}$ and Fabiola Angulo ${ }^{2}$ \\ ${ }^{1}$ Facultad de Ingeniería, Programa de Ingeniería Electrónica, Magma Ingeniería, Universidad del Magdalena, \\ Apartado Postal 2121630, Santa Marta, Colombia \\ ${ }^{2}$ Departamento de Ingeniería Eléctrica, Electrónica y Computación, Percepción y Control Inteligente, \\ Facultad de Ingeniería y Arquitectura, Universidad Nacional de Colombia (Sede Manizales), Campus La Nubia, \\ Bloque Q, Manizales 170003, Colombia
}

Correspondence should be addressed to Fabiola Angulo; fangulog@unal.edu.co

Received 17 October 2015; Accepted 16 November 2015

Academic Editor: Rongwei Guo

Copyright (C) 2015 J. A. Taborda and F. Angulo. This is an open access article distributed under the Creative Commons Attribution License, which permits unrestricted use, distribution, and reproduction in any medium, provided the original work is properly cited.

The aim of this paper is to describe and prove a new method to compute and control the basins of attraction in multistability scenarios and guarantee monostability condition. In particular, the basins of attraction are computed only using a submap, and the coexistence of periodic solutions is controlled through fixed-point inducting control technique, which has been successfully used until now to stabilize unstable periodic orbits. In this paper, however, fixed-point inducting control is used to modify the domains of attraction when there is coexistence of attractors. In order to apply the technique, the periodic orbit whose basin of attraction will be controlled must be computed. Therefore, the fixed-point inducting control is used to stabilize one of the periodic orbits and enhance its basin of attraction. Then, using information provided by the unstable periodic orbits and basins of attractions, the minimum control effort to stabilize the target periodic orbit in all desired ranges is computed. The applicability of the proposed tools is illustrated through two different coupled logistic maps.

\section{Introduction}

Complex bifurcation scenarios have been observed in nonlinear dynamic systems from virtually all areas of science, including a broad range of natural sciences, mechanical and electrical engineering, and economics and other areas of the social sciences [1-3]. Theoretical and applied researches have explained various bifurcation scenarios [4-6], and analytical, numerical, and experimental works have contributed to unraveling the complexity inherent to chaotic motion [3].

Coupled chaotic maps are a set of special discrete-time dynamical systems that can describe chemical, epidemiological, physiological, biological, or engineering systems [7]. Interesting nonlinear phenomena have been reported in them. For example, in $[8,9]$, coupled logistic maps were analyzed and new scenarios for transition to chaos were found via the creation and destruction of multilayered tori. In those papers, novel routes to chaos were described, and authors found that, depending on the coupling constant value, the system approaches different periodic attractors.

Control methods of coexisting attractors in multistability scenarios have been widely studied in the last decades. In [10], periodic signals were replaced by chaotic ones in order to eliminate multiple domains of attraction. In [11, 12], the influence of noise on preference and dominance of attractors in multistable systems was studied. In [13, 14], multistability was controlled using small periodic modulation of a system parameter. In [15], the basins of attraction (BA) were controlled using harmonic and stochastic modulation. In [16], an impulsive force was used in order to perturb one attractor of the system and to change its response such that the system response evolved to another attractor. In [17], control of multistability scenarios based on the selection of a particular attractor by periodic external modulation was presented. A complete report of control of multistability can be found in [18]. 
In this paper, we prove a different technique to control domains of attraction in multistability scenarios which is called Fixed-Point Inducting Controller (FPIC). The FPIC is a feed-forward controller that forces the system solution to evolve to an existing desired attractor which cannot be always reached for the uncontrolled case because of initial conditions. This technique was initially proposed in [19] and successfully applied in [20, 21]. In particular, in [21], experiments showed the good performance of the controlled system, where the FPIC was used as a second control loop. However, in all previous works published until now, FPIC has been only used to stabilize unstable periodic orbits.

The main contributions of this paper lie in numerical analysis and control design areas. The development of a novel methodology to compute and analyze the basins of attraction reinforced the numerical analysis. The methodology consists in decomposing the map of the system in $p$ submaps, where $p$ corresponds to the order of the periodic orbit to be controlled. To have the BA, the long-time response terms of this submap are depicted according to key colours. Apart from help to the control design, this way to compute the basin of attraction can be seen as the basins of attraction of period-1 orbits which are computed every sixth or fifth iteration for linear and nonlinear coupling, respectively, and the control goal can be thought of as the control of period-1 orbit. On the other hand, the methodology to design the controller based on FPIC technique belongs to control theory. When the proposed controller is used, the system is forced to evolve to a known periodic orbit that exists in the uncontrolled map. Moreover, by using the information obtained from bifurcation diagrams and basins of attraction, it is possible to compute the minimum control effort required to stabilize the target orbit in the defined region. In particular, the coexistence of periodic solutions in coupled logistic maps $[8,9,22]$ is controlled by widening the basin of attraction of a period- $p$ orbit that coexists with another one, and the minimum control effort is computed aided by the unstable period- 2 orbit for the linear coupling and by unstable period1 orbits for the nonlinear coupling case.

The paper is organized as follows. In Section 2, the coupled logistic maps are presented. The coexistence of period-6 and period-5 orbits is identified. In Section 3, the methodology to compute the basins of attraction is explained, and the procedure is applied to the linear and nonlinear coupling maps. In Section 4, the methodology to compute the controller is presented and applied to the considered systems and the minimum control effort required to guarantee monostabilization of the periodic orbit is computed. In Section 5, a brief discussion of the results is presented, and finally, in Section 6, the conclusions are given.

\section{Coupled Logistic Maps}

Coupled chaotic maps provide a source of bifurcation scenarios with nonlocal phenomena and coexistence of attractors. To design the proposed controller, two maps with different coupling mechanisms are chosen. For linear coupling map, there are two period- 6 orbits that coexist in a range of the parameter set. Similarly, for nonlinear coupling case, two period-5 orbits coexist.

2.1. Coupled Logistic Maps: Linear Coupling. This system is described by

$$
\begin{aligned}
& x(k+1)=f_{x}(k)+\epsilon(y(k)-x(k)), \\
& y(k+1)=f_{y}(k)+\epsilon(x(k)-y(k)),
\end{aligned}
$$

where $\epsilon \in(-2,2)$ is the coupling constant and $f_{z}(k)=$ $r z(1-z), r \in(1,4)$, is the parameter associated with the nonlinear part of (1). Since the system is symmetrical, there is an invariant line $\Delta$ and the restriction of the 2D map to $\Delta$ reduces it to a $1 \mathrm{D}$ map (the logistic map). The dynamics on this invariant set help us to study the dynamics and bifurcations of the $2 \mathrm{D}$ system. Moreover, symmetrical trajectories are generated by symmetrical initial conditions leading to symmetrical basins of attraction [22]. Novel routes to chaos through torus-breakdown mechanism of this system were reported in [9] and different dynamics were characterized in the parameter region given by $(\epsilon, r)$.

Figure 1 shows the dynamic behavior of (1) when $r$ is varied and two symmetrical initial conditions are considered. Note that a pair of coexisting period- 6 orbits are identified in these diagrams for $r \in[3.045,3.065]$, which makes the main difference between Figures 1(a) and 1(b). The dynamical behavior in the rest of the interval is very similar and other differences cannot be easily seen. Table 1 shows the specific values of the two coexisting period- 6 orbits. It can be observed that the solutions are mutually symmetrical in the sense that $(x, y)=(y, x)$.

2.2. Coupled Logistic Maps: Nonlinear Coupling. This system is defined by

$$
\left(\begin{array}{l}
x(k+1) \\
y(k+1)
\end{array}\right)=\left(\begin{array}{c}
f_{x}(k)+\epsilon\left(f_{y}(k)-f_{x}(k)\right) \\
f_{y}(k)+\epsilon\left(f_{x}(k)-f_{y}(k)\right)
\end{array}\right),
$$

where $f_{x}(k)$ and $f_{y}(k)$ were defined before, $\epsilon \in(-2,2)$, and $r \in(1,4)$. Figure 2 shows an interesting coexistence scenario for this system. Two mutually symmetrical period-5 cycles coexist in it when $\epsilon=1.5$ and $r \in[2.775,2.815]$.

\section{Methodology to Compute the Basins of Attraction}

In this section, a methodology to compute the basins of attraction (BA) is developed. By using this methodology, it is possible to find the minimum control effort required to stabilize a desired periodic orbit.

3.1. Computation of the Basins of Attractions in Linear Coupling Maps. The BA corresponds to the set of initial conditions whose long-time response (LTR) approaches the attractor. The first and second period- 6 orbits were defined in Table 1. All numerical results associated with the second period- 6 orbit are differentiated from the first one with an 
TABLE 1: Two coexisting period- 6 orbits for the linear coupling maps when $\epsilon=0.2725$ and $r=3.065$. For the sake of simplicity, the periodic orbit values are presented with 8 decimal digits.

\begin{tabular}{lc}
\hline First period-6 orbit & Second period-6 orbit \\
\hline$\left(x^{*}, y^{*}\right)_{1}=(0.79744636,0.20236605)$ & $\left(\bar{x}^{*}, \bar{y}^{*}\right)_{1}=(0.20236605,0.79744636)$ \\
$\left(x^{*}, y^{*}\right)_{2}=(0.33291675,0.65689339)$ & $\left(\bar{x}^{*}, \bar{y}^{*}\right)_{1}=(0.65689339,0.33291675)$ \\
$\left(x^{*}, y^{*}\right)_{3}=(0.76896860,0.60251974)$ & $\left(\bar{x}^{*}, \bar{y}^{*}\right)_{1}=(0.60251974,0.76896860)$ \\
$\left(x^{*}, y^{*}\right)_{4}=(0.49915797,0.77939325)$ & $\left(\bar{x}^{*}, \bar{y}^{*}\right)_{1}=(0.77939325,0.49915797)$ \\
$\left(x^{*}, y^{*}\right)_{5}=(0.84261193,0.45063017)$ & $\left(\bar{x}^{*}, \bar{y}^{*}\right)_{1}=(0.45063017,0.84261193)$ \\
$\left(x^{*}, y^{*}\right)_{6}=(0.29965625,0.86559446)$ & $\left(\bar{x}^{*}, \bar{y}^{*}\right)_{1}=(0.86559446,0.29965625)$ \\
\hline
\end{tabular}

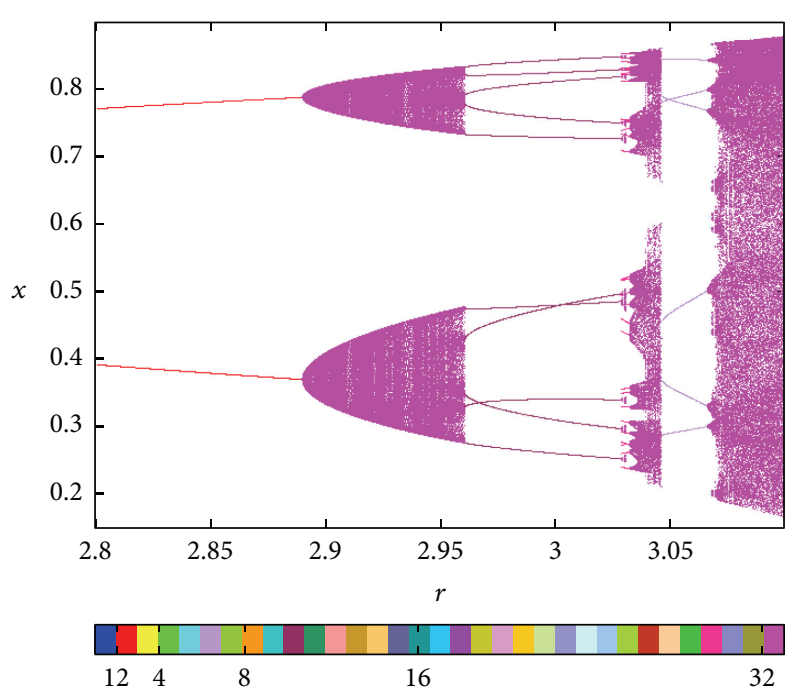

(a) $\left(x_{0}, y_{0}\right)=(0.1,0.5)$

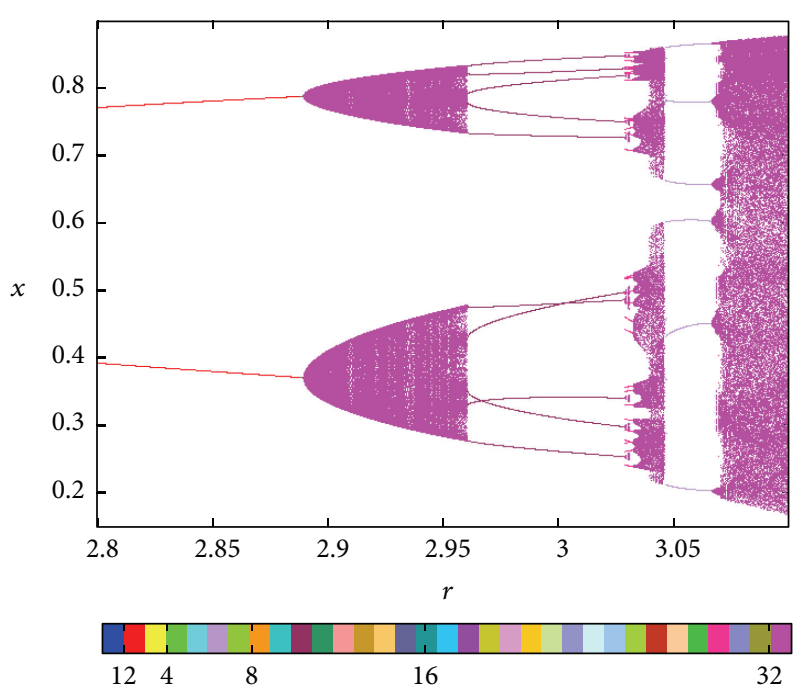

(b) $\left(x_{0}, y_{0}\right)=(0.5,0.1)$

FIGURE 1: Bifurcation diagrams of (1) with $\epsilon=0.2725$ and two symmetrical initial conditions.

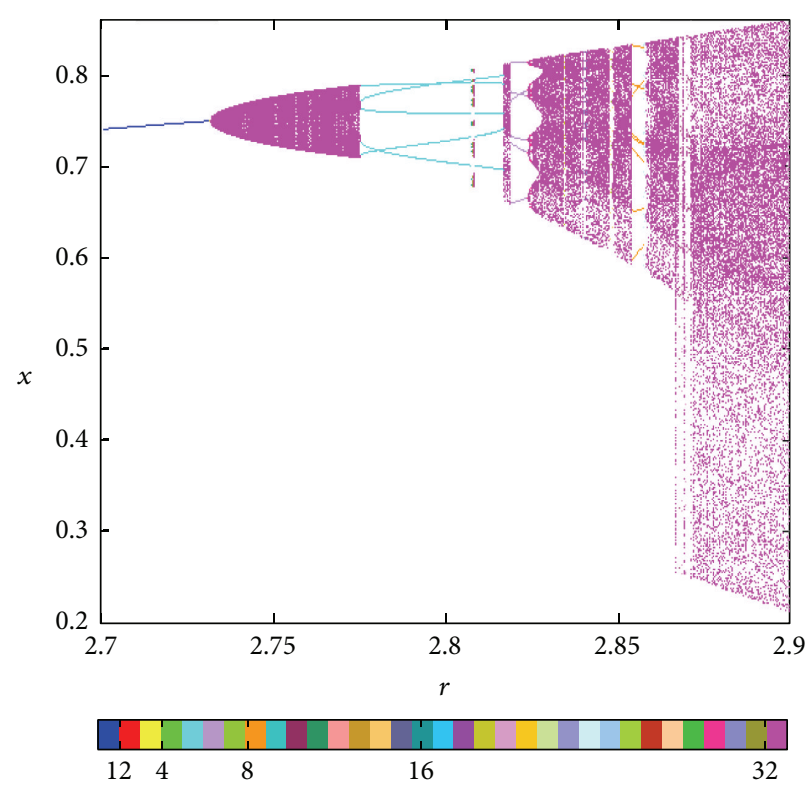

(a)

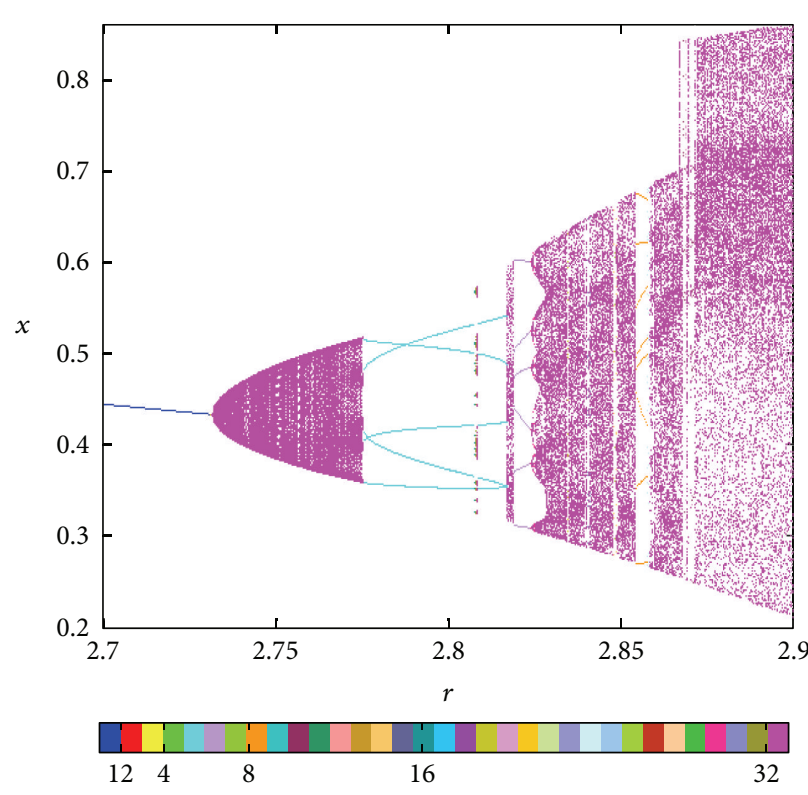

(b)

FIGURE 2: Bifurcation diagrams of (2) with $\epsilon=1.5$ and two symmetrical initial conditions. 


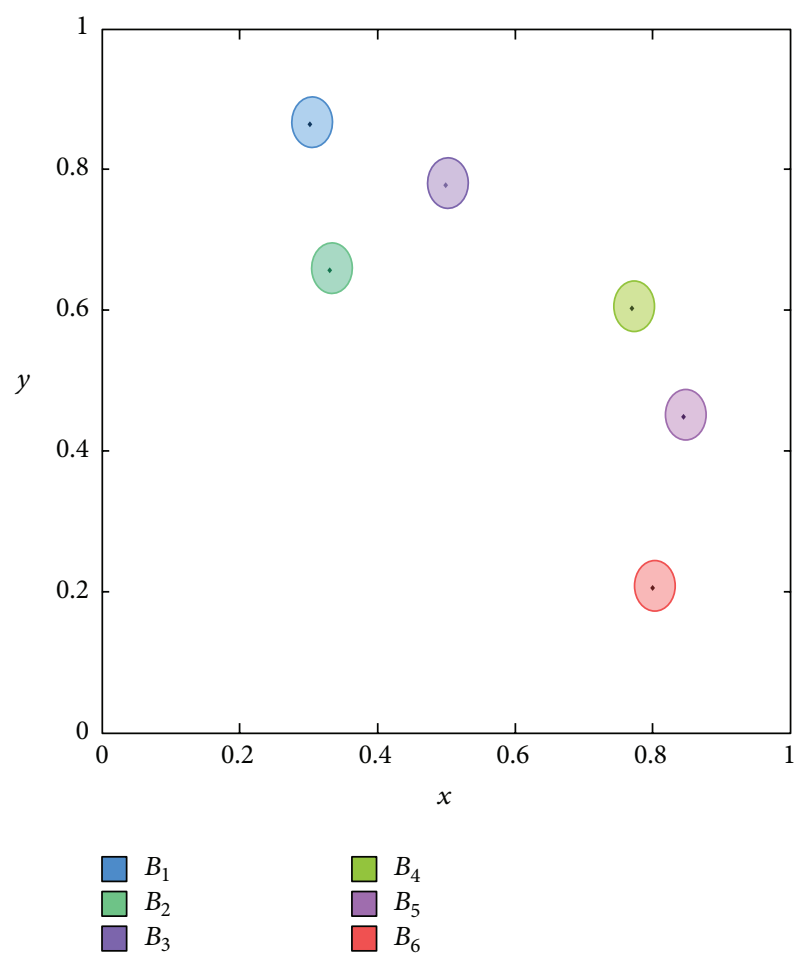

FIgURE 3: Colour key to compute the BA.

upper bar. In order to compute the BA and after applying FPIC control, we associate with each point $k$ of the first period- 6 orbit a section $B_{k}$ that is given by $\left|x_{\mathrm{LTR}}-x^{*}\right|<\delta$, such as what is illustrated in Figure 3. A similar procedure is developed to compute the BA of the second period- 6 orbit, whose sections are defined as $\bar{B}_{k}$.

To compute the LTR terms, we start from any initial condition inside the considered region $\left(0<x_{1}<1\right.$ and $\left.0<x_{2}<1\right)$ and compute its successive iterations until the solution approaches the attractor. The general map to describe the evolution of the system is $P$, and it is given by

$$
\begin{aligned}
P & :=\mathbf{x}(0) \longmapsto \mathbf{x}(1) \longmapsto \mathbf{x}(2) \longmapsto \mathbf{x}(3) \longmapsto \mathbf{x}(4) \\
& \longmapsto \mathbf{x}(5) \longmapsto \mathbf{x}(6) \longmapsto \mathbf{x}(7) \longmapsto \mathbf{x}(8) \longmapsto \mathbf{x}(9) \\
& \longmapsto \cdots \mathbf{x}(m)
\end{aligned}
$$

where $m$ is high enough. The map $P$ is decomposed in six submaps $P_{i}$. Each $P_{i}$ is formed by the set of points given by $\mathbf{x}(i-1+6 j)$, where $i=1 \cdots 6$ and $j \in \mathbb{Z}^{+}+\{0\}$. If the LTR terms of $P_{i}$ lie inside some $B_{k}$ (or $\bar{B}_{k}$ when the BA for the second period- 6 orbit is computed), it is coloured according to the colour key displayed in Figure 3. If the LTR terms of $P_{i}$ lie outside these regions, then it is gray coloured. This procedure is repeated for a lot of initial conditions.

The BA of the first period- 6 orbit (second period-6 orbit) can be computed and depicted only using $P_{i}\left(\bar{P}_{i}\right)$ submaps for a fixed $i$, and its diagram may have up to seven colors. The gray coloured points correspond to the set of initial conditions whose LTR terms diverge from the first period- 6 orbit (second period- 6 orbit), and the other points (depicted with six different colors according to Figure 3) correspond to the initial conditions whose LTR terms lie inside some $B_{k}\left(\bar{B}_{k}\right)$ region. The BA computed for the first period- 6 orbit (second period- 6 orbit) in this way is noted as $\mathrm{BA}\left(P_{i}\right)\left(\mathrm{BA}\left(\bar{P}_{i}\right)\right)$. Figure $4(\mathrm{a})$ shows the diagram associated with $\mathrm{BA}\left(P_{1}\right)$. All $\mathrm{BA}\left(P_{i}\right)$ have the same information but are coloured in different way. Figure $4(\mathrm{~b})$ shows $\mathrm{BA}\left(\bar{P}_{1}\right)$. These $\mathrm{BA}$ let us compute the minimum control effort required to stabilize the periodic orbits.

\subsection{Computation of the Basins of Attraction for Nonlinear} Coupling Maps. The procedure explained before is applied to the nonlinear coupling maps. $\mathrm{BA}\left(P_{i}\right)$ and $\mathrm{BA}\left(\bar{P}_{i}\right)$ of the two period-5 orbits are also symmetrical. Figures $5(\mathrm{a})$ and 5(b) show $\mathrm{BA}\left(P_{1}\right)$ and $\mathrm{BA}\left(\bar{P}_{1}\right)$, respectively. The remaining diagrams $\mathrm{BA}\left(P_{i}\right)$ and $\mathrm{BA}\left(\bar{P}_{i}\right)$ for $i=2 \cdots 5$ have similar behavior. As we said before, all $\mathrm{BA}\left(P_{i}\right)$ carry the same information but are coloured in different way.

\section{Methodology to Control the Basins of Attraction}

In this section, domains of attraction in coexistence scenarios are controlled using FPIC technique. This technique has been proven to stabilize unstable and chaotic systems [19-21]. In those papers, analytical or numerical values of the steadystate control inputs were needed to guarantee the stabilization of the equilibrium point because FPIC was used as a second control loop. On the contrary, in this work, FPIC technique is used to control the BA in coupled logistic maps to guarantee stabilization of period-6 (for linear coupling) and period5 (for nonlinear coupling) orbits, using the values of the periodic orbit to be controlled.

\subsection{Fixed-Point Inducting Control}

Theorem 1. Consider a discrete-time system

$$
\mathbf{z}(k+1)=\mathbf{g}(\mathbf{z}(k)) .
$$

It is assumed that period-p solutions coexist in the system and two or more of them are stable. A stable period-p solution is noted in a compact form as $\mathbf{z}^{*}$, and it is composed of $p$ points such that it can be expressed as

$$
\begin{gathered}
\mathbf{z}^{*}=\left\{z_{1}^{*}, z_{2}^{*}, \ldots, z_{p}^{*}\right\}=\left\{\left(x_{1}^{*}, x_{2}^{*}, \ldots, x_{n}^{*}\right)_{1},\right. \\
\left.\left(x_{1}^{*}, x_{2}^{*}, \ldots, x_{n}^{*}\right)_{2}, \ldots,\left(x_{1}^{*}, x_{2}^{*}, \ldots, x_{n}^{*}\right)_{p}\right\} .
\end{gathered}
$$

The objective of the controller is to expand the domain of attraction of a given period-p orbit, by forcing the system to evolve to this specific solution. Equation (6) shows the control law based on FPIC, with $E$ as the control parameter. This 


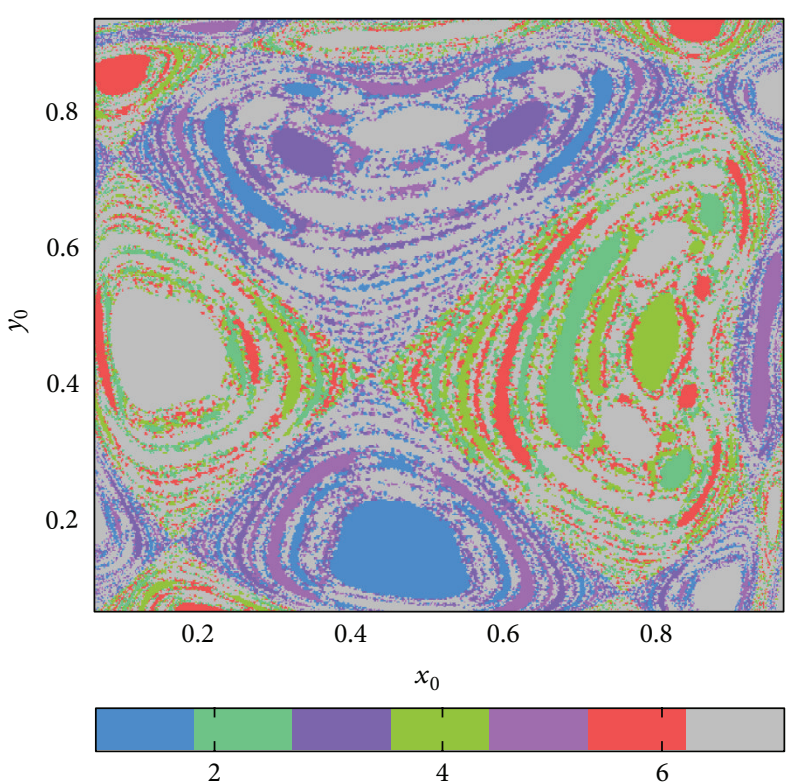

(a)

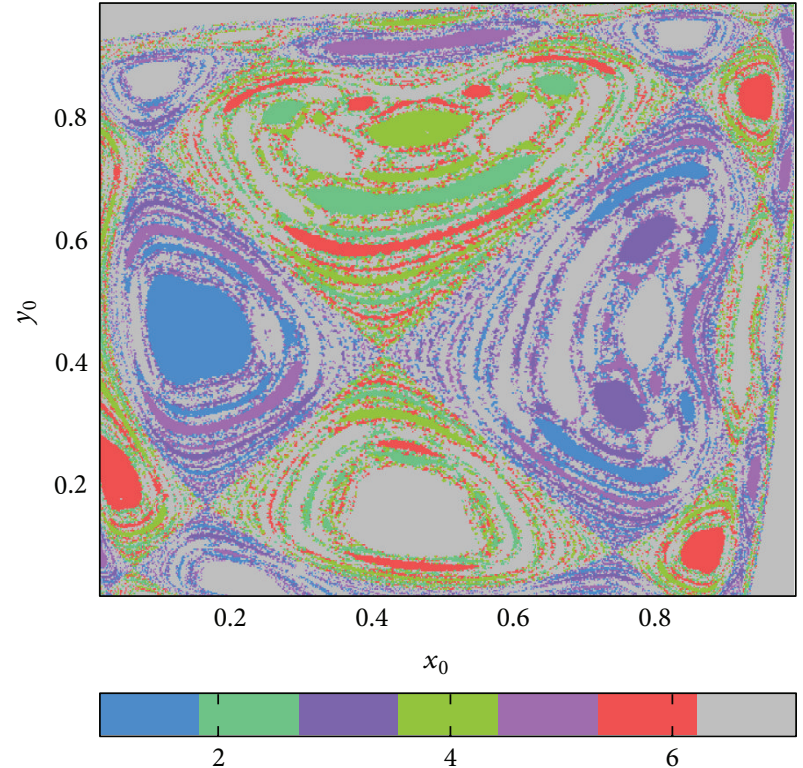

(b)

Figure 4: (a) BA( $\left.P_{1}\right)$. (b) $\mathrm{BA}\left(\bar{P}_{1}\right) \cdot r=3.065$ and $\epsilon=0.2725$.
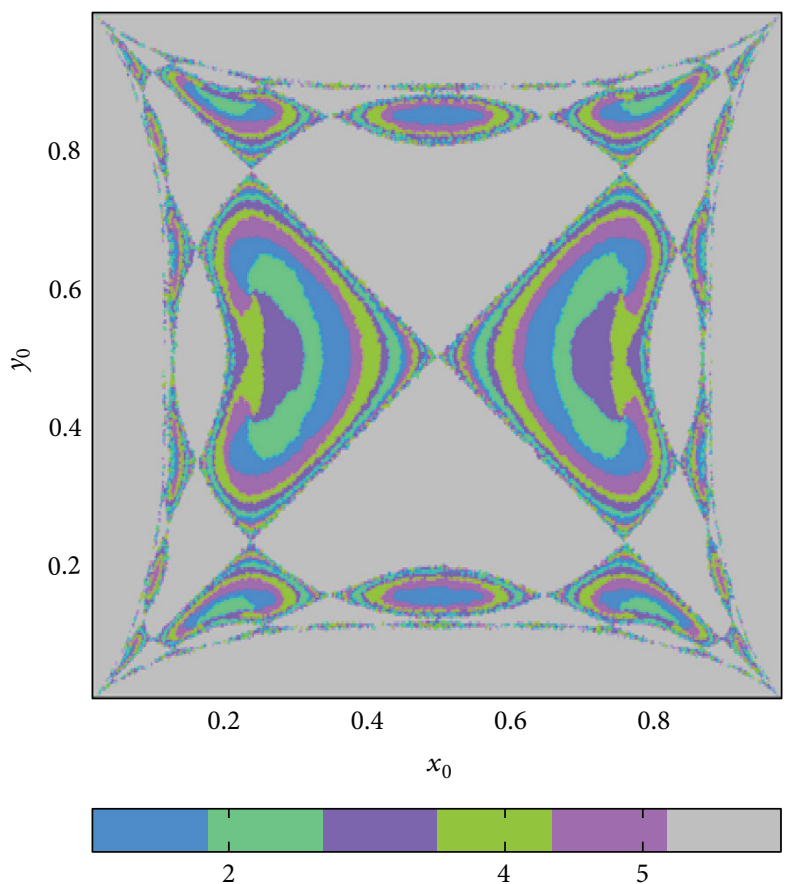

(a)

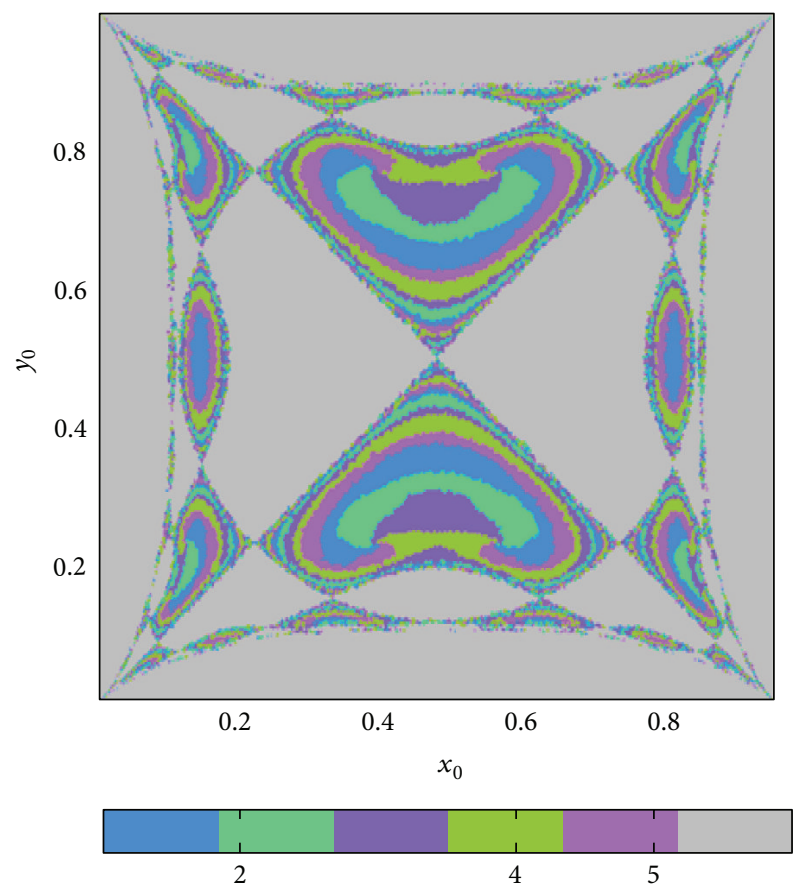

(b)

Figure 5: (a) $\mathrm{BA}\left(P_{1}\right)$. (b) $\mathrm{BA}\left(\bar{P}_{1}\right) \cdot r=2.8$ and $\epsilon=1.5$.

controller stabilizes the target periodic orbit and expands its attraction domain as E increases. Hence,

$$
\begin{aligned}
& \mathbf{z}(k+1) \\
& \quad= \begin{cases}\frac{1}{E+1} \mathbf{g}(\mathbf{z}(k))+\frac{E}{E+1} \mathbf{z}_{j}^{*} & \text { if }(k \bmod p)=0 \\
\mathbf{g}(\mathbf{z}(k)) & \text { if }(k \bmod p) \neq 0,\end{cases}
\end{aligned}
$$

where $j$ is defined depending on the analysis of the controlled system.

Proof. The periodic orbits of the controlled system are the same as the uncontrolled one. This is because after $p$ iterations $\mathbf{g}\left(z_{j}^{*}\right)=z_{j}^{*}$ (by definition of periodic orbit) and thus considering only the upper part of (6), it can be seen that the periodic 
orbits remain unchanged. Hence, when $E$ is high enough, the controller transfers the state instantly to some $B_{k}$ when $k \bmod p=0$, irrespective of the state value. In this way, the new state (that is near to the periodic solution) is the initial condition for the next iteration obtaining convergence to the periodic solution because the periodic orbit is stable.

Remarks. (i) From a practical point of view, the idea is not to fix $E$ to a high value because the control effort increases as $E$ does. To control systems whose initial conditions are away from the periodic orbit can lead to oversizing of the control effort. For example, for the systems analyzed in this work, the solution quickly diverges when the initial conditions are outside the considered region and the controller cannot be used.

(ii) The minimum value of $E$ that should be put in (6) can be found based on numerical analysis, where the unstable period orbits that coexist in the dynamical system play an important role.

4.2. Control of Period-6 Orbits in Linear Coupling Maps. Let us consider the map defined by (1) with $r=3.065$ and $\epsilon=$ 0.2725 . We will stabilize the second period -6 orbit using FPIC control. In order to achieve the goal, we use the second point $\left(\bar{x}^{*}, \bar{y}^{*}\right)_{2}$ as the target control; that is, $\overline{\mathbf{x}}_{2}^{*}:=(0.6569,0.3329)$. Taking into account (6), the linear coupling map is perturbed every sixth iteration according to the control law defined by

$$
\left(\begin{array}{l}
x(k+1) \\
y(k+1)
\end{array}\right)= \begin{cases}\frac{1}{E+1}\left(\begin{array}{l}
f_{x}(k)+\varepsilon(y(k)-x(k)) \\
f_{y}(k)+\varepsilon(x(k)-y(k))
\end{array}\right)+\frac{E}{E+1}\left(\begin{array}{l}
\bar{x}^{*} \\
\bar{y}^{*}
\end{array}\right) & \text { if }(k \bmod 6)=0 \\
\left(\begin{array}{l}
f_{x}(k)+\varepsilon(y(k)-x(k)) \\
f_{y}(k)+\varepsilon(x(k)-y(k))
\end{array}\right) & \text { if }(k \bmod 6) \neq 0 .\end{cases}
$$

Figure 6 shows $\mathrm{BA}\left(\bar{P}_{1}\right)$ diagram for different $E$ values. Colors were assigned as defined above. The elements of $\mathrm{BA}\left(\bar{P}_{1}\right)$ that tends to $\bar{B}_{2}$ increase as parameter $E$ increases. Comparing Figures 4(b) and 6, it can be seen that the green coloured region enhances forming clear narrow contours. Now, the BA is only formed by two sets of points. The first one includes all points whose LTR terms end in $\bar{B}_{2}$ which are green coloured. The second one includes all points whose LTR terms are not inside any $\bar{B}_{i}$ section for $i=1,2,3,4,5,6$, which are gray coloured. This implies that no long-time term of the submap $\bar{P}_{1}$ lies in $\bar{B}_{i}$ section, for $i=1,3,4,5,6$. The green contours expand as $E$ increases, widening the BA and diminishing the gray coloured parts.

Figure 7 shows $\mathrm{BA}\left(P_{1}\right)$ when $\mathbf{x}_{2}^{*}:=(0.3329,0.6569)$ is used as the reference in the FPIC controller. The same values of $E$ have been used.

4.2.1. Selection of Minimum Control Effort. The minimum control effort $E_{\mathrm{cr}}$ is defined as the minimum value of $E$ that forces the system to evolve to the controlled orbit. The selection of $E_{\mathrm{cr}}$ can be computed by studying the behavior of unstable periodic orbits (UPOs) around the periodic orbits.

The metamorphosis of controlled $\mathrm{BA}\left(\bar{P}_{1}\right)$ or $\mathrm{BA}\left(P_{1}\right)$ is influenced by two unstable period- 2 solutions of the system that are schematized in Figure 8. The fixed points of these period-2 orbits are named $V_{1}, V_{2}, V_{3}$, and $V_{4}$.
For the controlled system, the points $V_{i}$ are hard to cover by the expansion of $\mathrm{BA}\left(P_{1}\right)$. These points are always on the frontiers between the green and gray regions as it is shown in Figure 9. To find $E_{\mathrm{cr}}$, we consider $\Delta_{i}$ region around each point $V_{i} . E_{\mathrm{cr}}$ is the minimum value such that all points in $\Delta_{i}^{0}$ regions converge to $V_{i}$ points. Figure 9 shows examples of $\Delta_{i}$ regions when $E=1.5$. The neighborhood near $V_{i}$ requires more control effort to converge to $B_{2}$.

$\Delta_{i}$ can be expressed as $\left(\Delta_{x}, \Delta_{y}\right):=\left(v_{x}+\delta \sin (\theta), v_{y}+\right.$ $\delta \cos (\theta))$, where $\delta$ is fixed to a small value and $\theta$ is varied in the range $[0,2 \pi]$. Figure 10 illustrates the behavior of $\mathrm{BA}\left(P_{1}\right)$ around $\Delta_{i}$ when $E$ is varied. The convergence of all points to $\Delta_{i}$ is reached when $E_{\mathrm{cr}} \approx 1.78$.

4.2.2. Selection of the Reference. Similar results can be obtained if we choose other equilibrium points to perturb the map $P_{i}$. Table 3 summarizes the control effort requirements. Note that the critical control effort $E_{\mathrm{cr}}$ varies depending on the used reference. Coexistence phenomenon can be controlled with less effort if $\left(x^{*}, y^{*}\right)=(0.8426,0.4506)$ or $\left(\bar{x}^{*}, \bar{y}^{*}\right)=(0.4506,0.8426)$ are used to control the map.

4.3. Control of Period-5 Orbits in Nonlinear Coupling Maps. Now, we control two coexisting period-5 cycles of (2) when $r=2.8$ and $\epsilon=1.5$. The reference is introduced every fifth iteration as is presented in

$$
\left(\begin{array}{l}
x(k+1) \\
y(k+1)
\end{array}\right)= \begin{cases}\frac{1}{E+1}\left(\begin{array}{l}
f_{x}(k)+\varepsilon\left(f_{y}(k)-f_{x}(k)\right) \\
f_{y}(k)+\varepsilon\left(f_{x}(k)-f_{y}(k)\right)
\end{array}\right)+\frac{E}{E+1}\left(\begin{array}{l}
\bar{x}^{*} \\
\bar{y}^{*}
\end{array}\right) & \text { if }(k \bmod 5)=0 \\
\left(\begin{array}{l}
f_{x}(k)+\varepsilon\left(f_{y}(k)-f_{x}(k)\right) \\
f_{y}(k)+\varepsilon\left(f_{x}(k)-f_{y}(k)\right)
\end{array}\right) & \text { if }(k \bmod 5) \neq 0 .\end{cases}
$$


TABLE 2: Two coexisting period-5 orbits for nonlinear coupling maps when $\epsilon=1.5$ and $r=2.8$. For the sake of simplicity, the periodic orbit values are presented with 8 decimal digits.

\begin{tabular}{lc}
\hline First period-5 orbit & Second period-5 orbit \\
\hline$\left(x^{*}, y^{*}\right)_{1}=(0.79175845,0.35258819)$ & $\left(\bar{x}^{*}, \bar{y}^{*}\right)_{1}=(0.35258819,0.79175845)$ \\
$\left(x^{*}, y^{*}\right)_{2}=(0.72790518,0.37290574)$ & $\left(\bar{x}^{*}, \bar{y}^{*}\right)_{2}=(0.37290574,0.72790518)$ \\
$\left(x^{*}, y^{*}\right)_{3}=(0.70487469,0.50446287)$ & $\left(\bar{x}^{*}, \bar{y}^{*}\right)_{3}=(0.50446287,0.70487469)$ \\
$\left(x^{*}, y^{*}\right)_{4}=(0.75867944,0.52373859)$ & $\left(\bar{x}^{*}, \bar{y}^{*}\right)_{4}=(0.52373859,0.75867944)$ \\
$\left(x^{*}, y^{*}\right)_{5}=(0.79131428,0.41974570)$ & $\left(\bar{x}^{*}, \bar{y}^{*}\right)_{5}=(0.41974570,0.79131428)$ \\
\hline
\end{tabular}

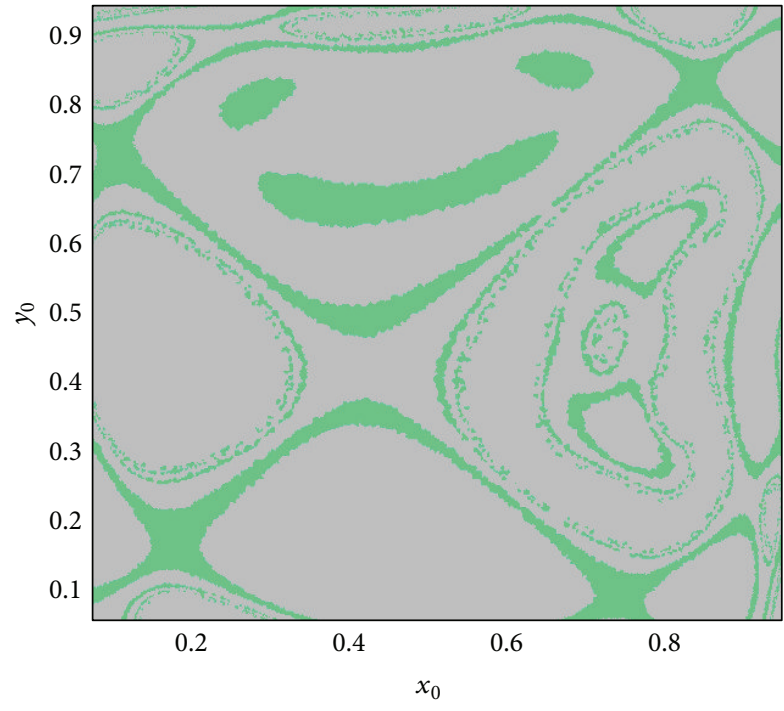

(a)

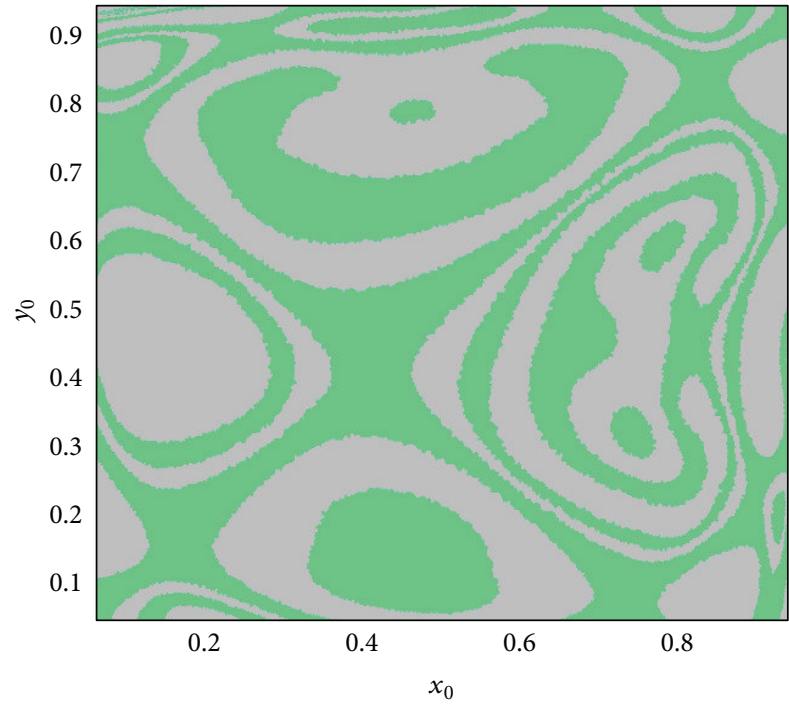

(b)

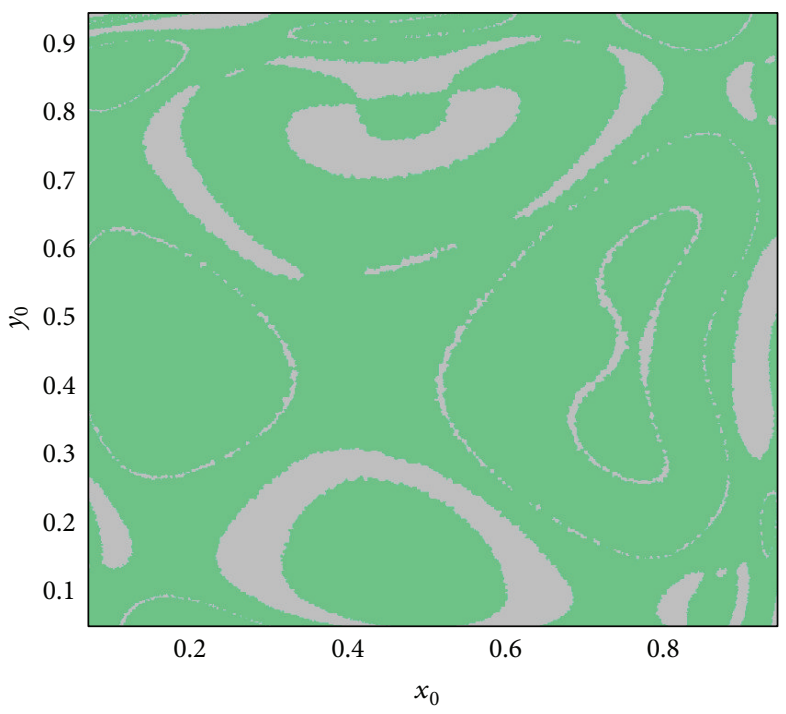

(c)

Figure 6: (a) $\mathrm{BA}\left(\bar{P}_{1}\right)$ for $E=0.85$. (b) $\mathrm{BA}\left(\bar{P}_{1}\right)$ for $E=1.5$. (c) $\mathrm{BA}\left(\bar{P}_{1}\right)$ for $E=1.7 . r=3.065$ and $\epsilon=0.2725$ in all cases.

According to Table 2 , we use $\left(\bar{x}^{*}, \bar{y}^{*}\right)_{1}:=(0.3526,0.7918)$ to control the system. Figure 11 presents $\mathrm{BA}\left(\bar{P}_{1}\right)$ of the controlled system when three different values of $E$ are considered.

Selection of Minimum Control Effort. As in the previous case, the minimum control effort $E_{\mathrm{cr}}$ can be computed by studying the behavior of UPOs around the periodic orbits. Figure 12 shows an illustrative sketch of bifurcation scenario of the uncontrolled system. The unstable period-1 orbits are noted as $N_{1}$ and $N_{2}$. The metamorphosis of the controlled maps $\mathrm{BA}\left(\bar{P}_{i}\right)$ is influenced by unstable period-1 solutions of the system. We use points $N_{1}$ and $N_{2}$ to compute $E_{\text {cr }}$ in each case. 


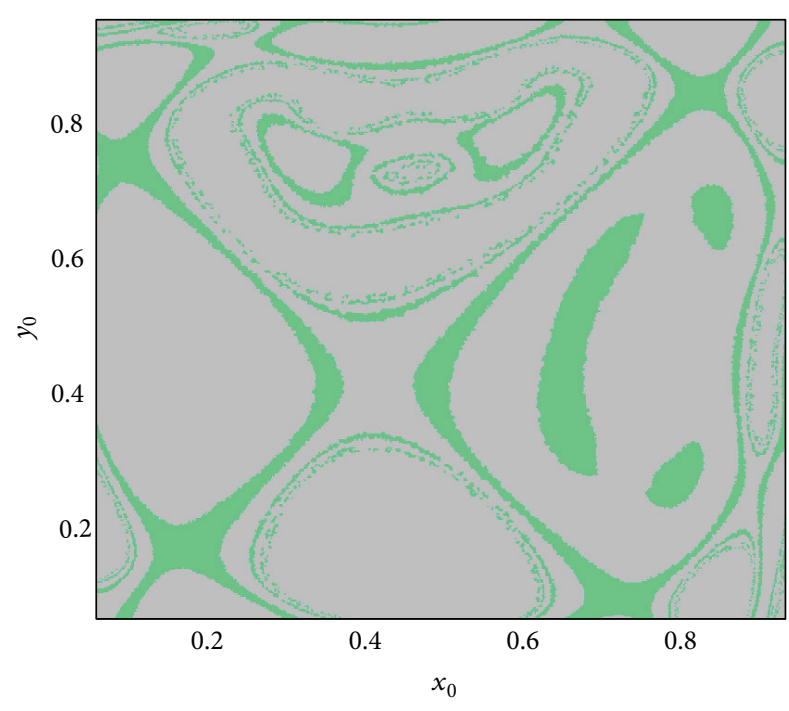

(a)

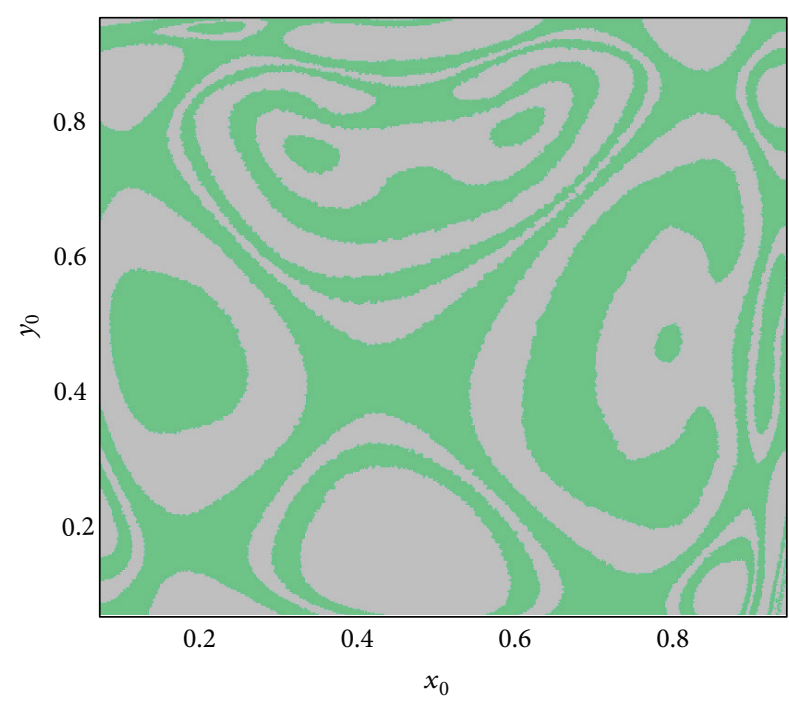

(b)

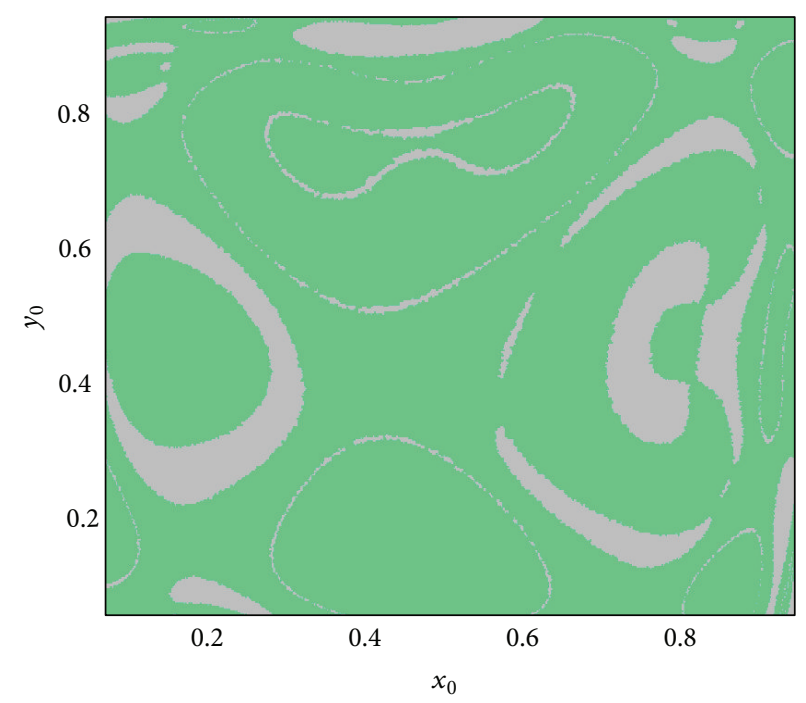

(c)

Figure 7: (a) $\mathrm{BA}\left(P_{1}\right)$ for $E=0.85$. (b) $\mathrm{BA}\left(P_{1}\right)$ for $E=1.5$. (c) $\mathrm{BA}\left(P_{1}\right)$ for $E=1.7 . r=3.065$ and $\epsilon=0.2725$ for all cases.

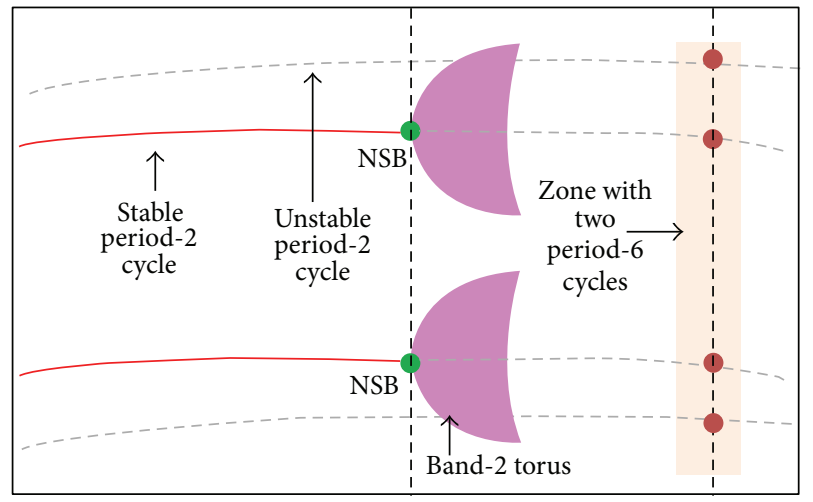

FIGURE 8: Sketch of unstable period-2 solutions $V_{i}$ in coexistence zone.
Table 4 presents the minimum value of parameter $E$ to control coexistence phenomenon. All initial conditions belonging to the considered region converge to $\overline{\mathbf{x}}^{*}$ when $E>E_{\mathrm{cr}}$. The lowest control effort that avoids coexistence phenomenon is reached when the map is controlled with the first point of the periodic orbit. If we induce the control using other points of the periodic orbit, then we need a greater control effort. Figure 13 shows the symmetrical behavior between $\mathrm{BA}\left(P_{1}\right)$ and $\operatorname{BA}\left(\bar{P}_{1}\right)$ (compare with Figure 11). Critical control effort required in both cases has the same value.

\section{Discussion}

The results presented above give rise to a new approach for coexistence control in multistable systems. The proposed 


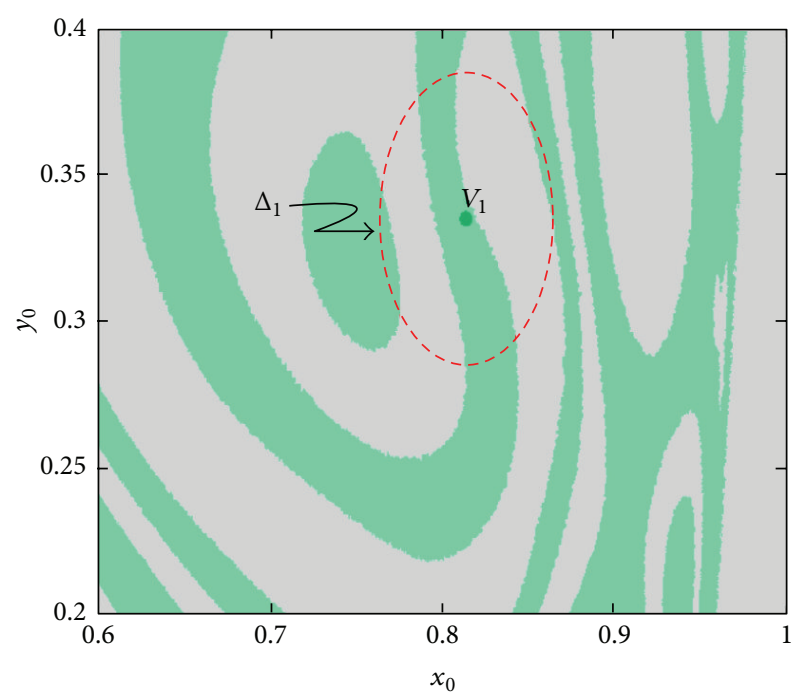

(a)

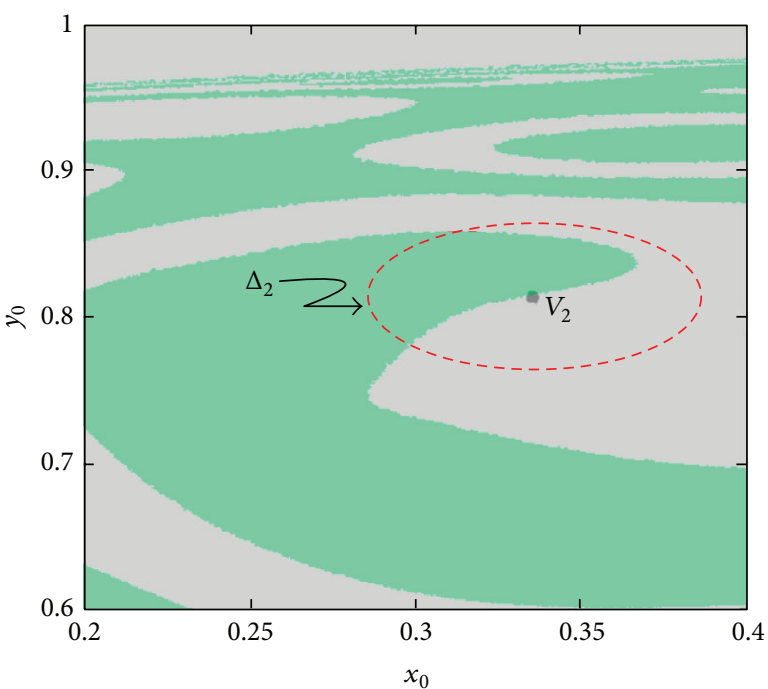

(b)

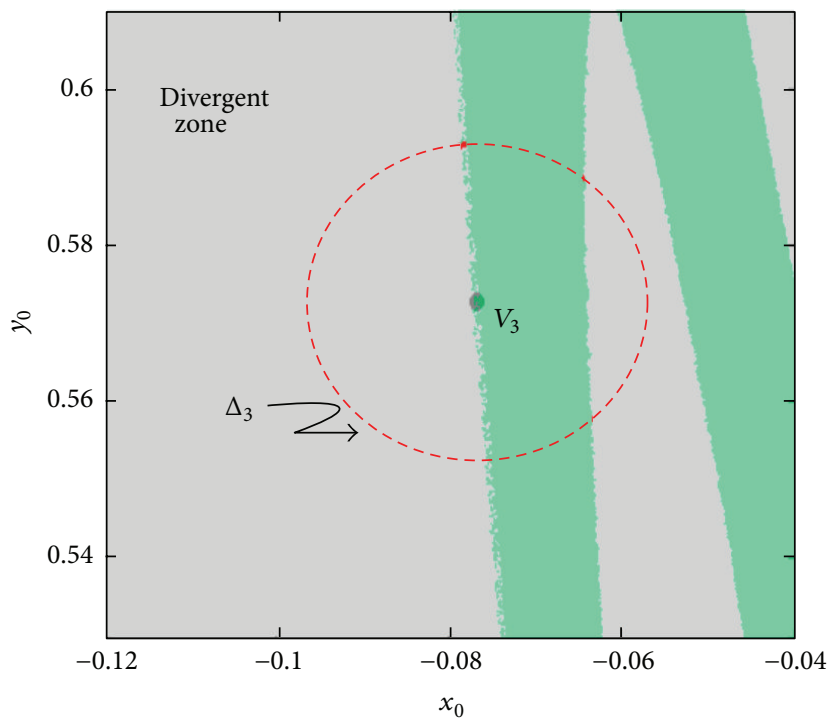

(c)

FIGURE 9: $V_{i}$ points and $\Delta_{i}$ sections in controlled linear map with $E=1.5$. (a) $V_{1}=(0.81326798,0.33518226)$. (b) $V_{2}=(0.33518226$, $0.81326798)$. (c) $V_{3}=(-0.07696298,0.57288468)$.

TABLE 3: Summary of FPIC requirements in coexistence control of two period- 6 cycles.

\begin{tabular}{lccc}
\hline Maps & $\mathbf{x}^{*}$ & $\overline{\mathbf{x}}^{*}$ & $E_{\text {cr }}$ \\
\hline$P_{1}, \bar{P}_{1}$ & $(0.7974,0.2023)$ & $(0.2023,0.7974)$ & 3.371 \\
$P_{2}, \bar{P}_{2}$ & $(0.3329,0.6568)$ & $(0.6568,0.3329)$ & 1.774 \\
$P_{3}, \bar{P}_{3}$ & $(0.7689,0.6025)$ & $(0.6025,0.7689)$ & 2.507 \\
$P_{4}, \bar{P}_{4}$ & $(0.4991,0.7793)$ & $(0.7793,0.4991)$ & 1.492 \\
$P_{5}, \bar{P}_{5}$ & $(0.8426,0.4506)$ & $(0.4506,0.8426)$ & 1.258 \\
$P_{6}, \bar{P}_{6}$ & $(0.2996,0.8655)$ & $(0.8655,0.2996)$ & 4.112 \\
\hline
\end{tabular}

methodology has significant differences with other methods reported in the literature, some of which are commented on below.
TABLE 4: Summary of FPIC requirements in coexistence control of two period-5 cycles.

\begin{tabular}{lccc}
\hline Maps & $\mathbf{x}^{*}$ & $\overline{\mathbf{x}}^{*}$ & $E_{\mathrm{cr}}$ \\
\hline$P_{1}, \bar{P}_{1}$ & $(0.7918,0.3526)$ & $(0.3526,0.7918)$ & 0.6099 \\
$P_{2}, \bar{P}_{2}$ & $(0.7279,0.3729)$ & $(0.3729,0.7279)$ & 0.6873 \\
$P_{3}, \bar{P}_{3}$ & $(0.7049,0.5045)$ & $(0.5045,0.7048)$ & 1.2312 \\
$P_{4}, \bar{P}_{4}$ & $(0.7588,0.5237)$ & $(0.5237,0.7588)$ & 1.1892 \\
$P_{5}, \bar{P}_{5}$ & $(0.7913,0.4197)$ & $(0.4197,0.7913)$ & 0.7504 \\
\hline
\end{tabular}

(i) The Applicability of the Method. Our process is sequential, systematic, and applicable to any multistable system, even though we use low-dimensional discrete maps as illustrative 


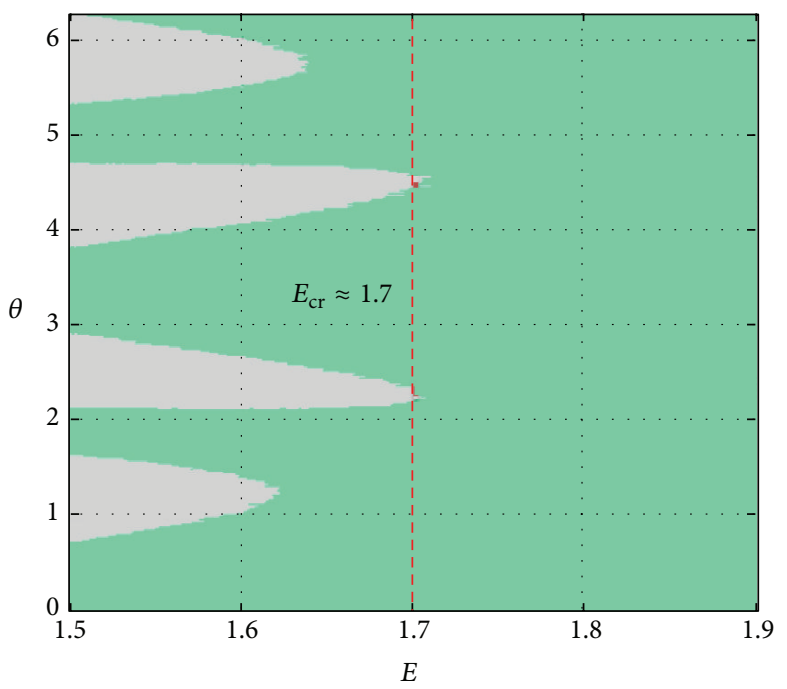

(a) $V_{1}$

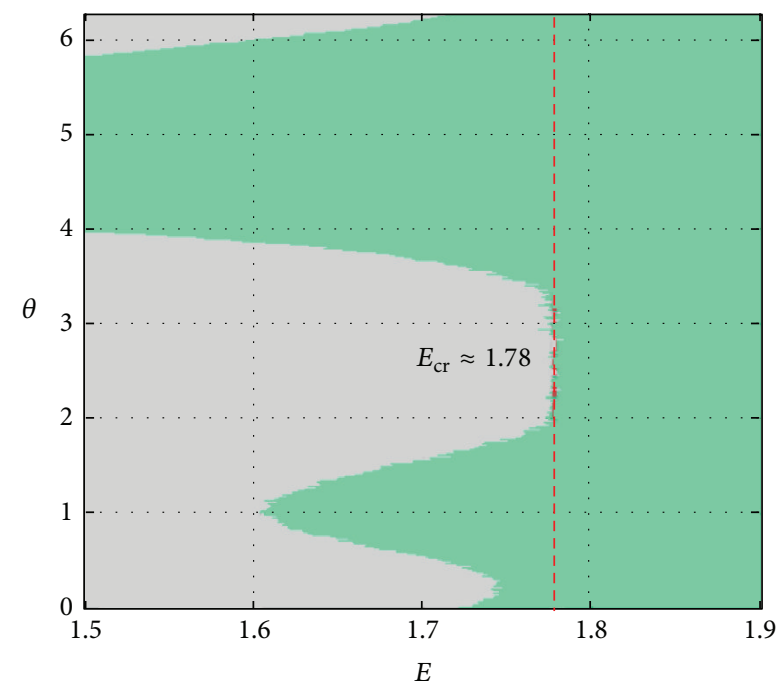

(b) $V_{2}$

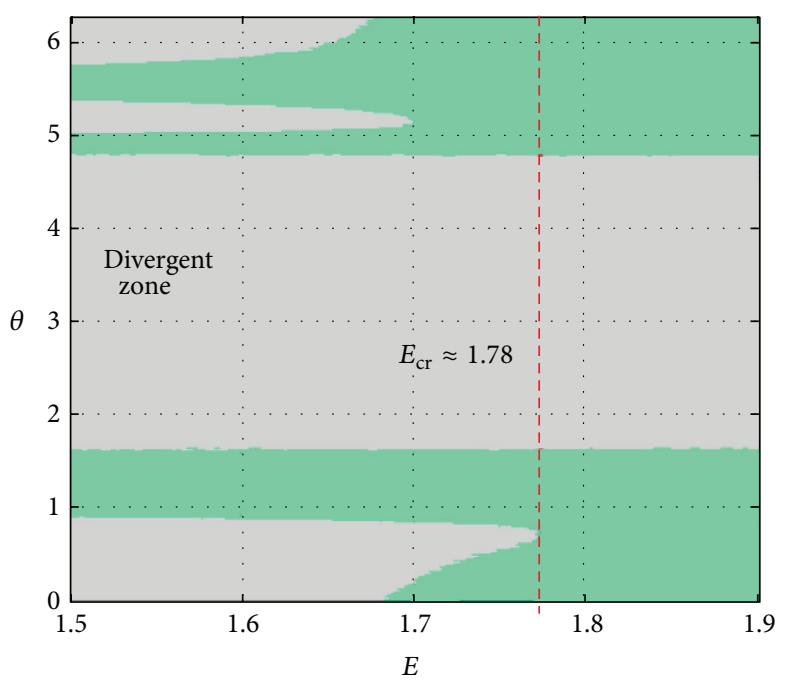

(c) $V_{3}$

Figure 10: Selection of critical value $E_{\mathrm{cr}}$. Diagram $E$ versus $\theta$ to select minimum value of $E$ that guarantees total convergence of $P_{1}$ to $x^{*}=$ 0.3329 and $y^{*}=0.6568$.

examples. One of the most common methods to control multistability, which is based on slow harmonic modulation, has restrictions on its application because the attractor to be destroyed should be a focus type [23].

(ii) The Requirement of Dynamic Behavior Knowledge. Fixedpoint inducting control needs to know an approximate value of the periodic orbit to be stabilized, while all nonfeedback methods do not require a priori information of the system to be controlled [18]. However, this is not a major problem due to the simplicity of the required information and the multiple ways to obtain it, by either theoretical, empirical, or experimental sources. (iii) The Control Scheme, the Choice of the Attractor, and the Number of Parameters to Be Tuned. The most representative methods for multistability control can be classified into three categories: nonfeedback, feedback, and stochastic schemes [18]. We propose a feed-forward control configuration which has not been widely exploited. Particularly, the control law based on FPIC criterion has advantages with respect to the selection of the target orbit and the parameters setting. For example, when the coexistence control is realized by parameter modulation, the general form given by $p=p_{0}+$ $p_{c} \sin \left(2 \pi f_{c} t+\psi_{0}\right)$ has four parameters to be tuned [24]; then, the selection of a particular attractor depends on the right choice of these parameters. In contrast, to successfully apply 


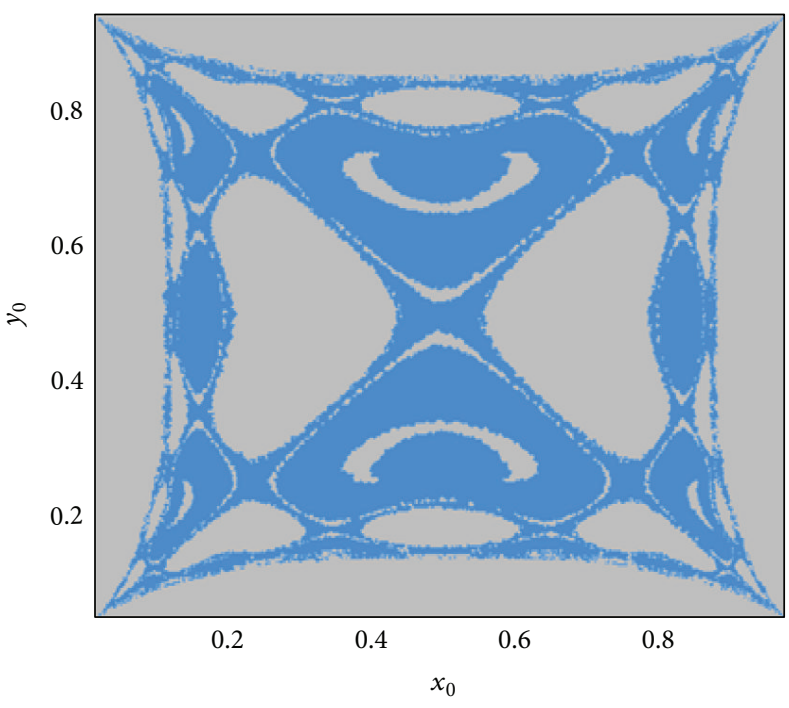

(a)

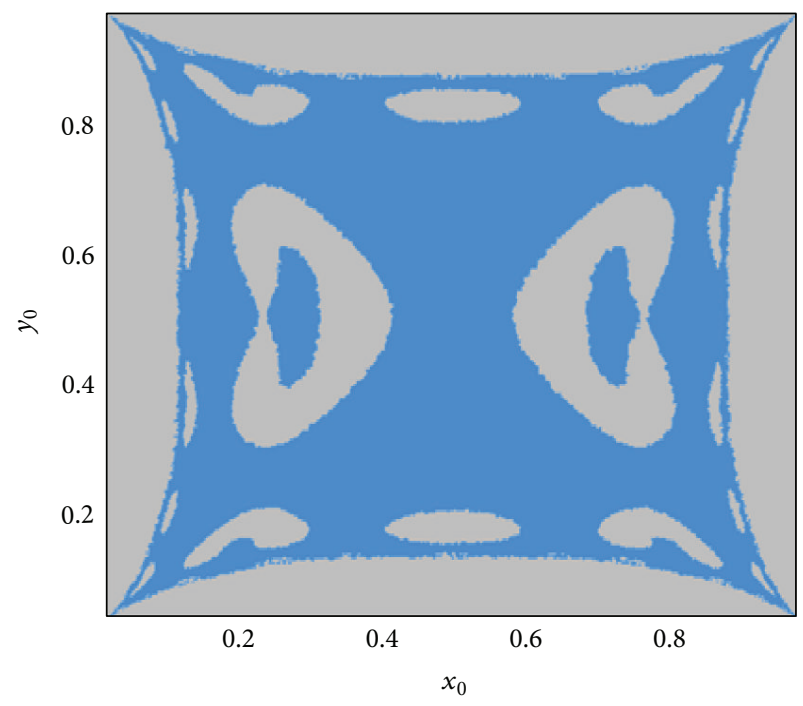

(b)

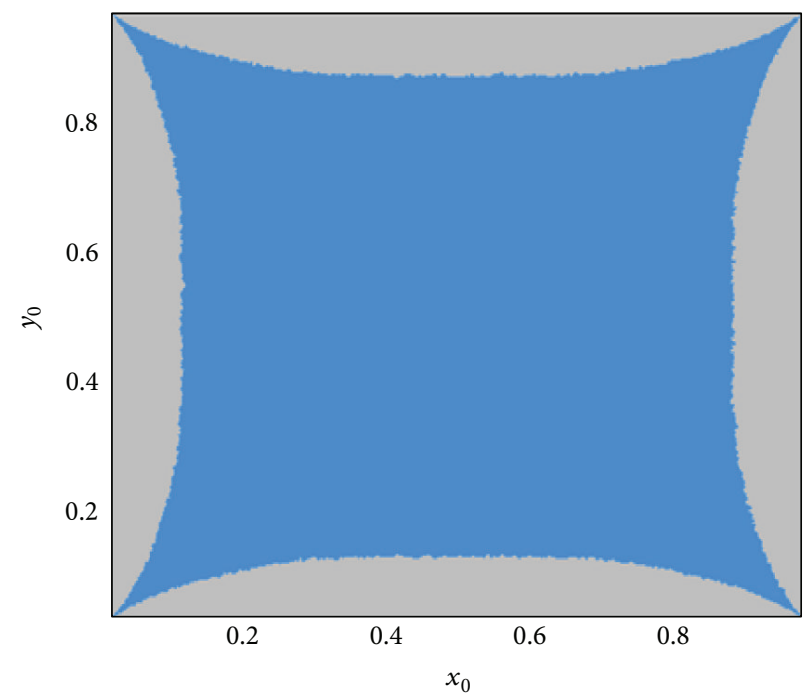

(c)

Figure 11: $\mathrm{BA}\left(\bar{P}_{1}\right)$ for $E=0.25$. (b) $\mathrm{BA}\left(\bar{P}_{1}\right)$ for $E=0.45$. (c) $\mathrm{BA}\left(\bar{P}_{1}\right)$ for $E=0.65 . r=2.8$ and $\epsilon=1.5$ for all cases.

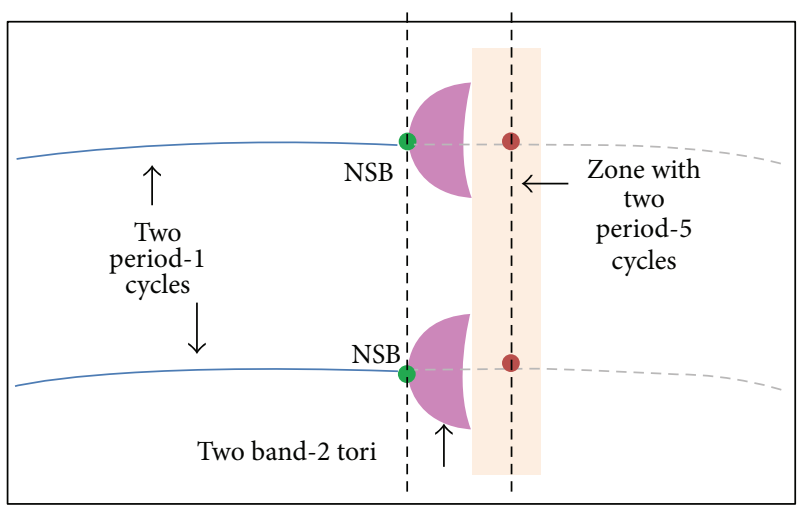

FIgURE 12: Sketch of unstable period-1 solutions $N_{i}$ in coexistence zone.
FPIC control only it is needed to tune one parameter and to know the target orbit.

\section{Conclusions}

The coexistence of periodic solutions has been controlled using FPIC technique and the required control effort to obtain stabilization of the periodic orbits was computed.

It has been proven that FPIC controller can be used to control BA. FPIC requires virtually no information about the system; just approximated knowledge of a periodic orbit is needed. Thus, this is a good control technique if the system is only known approximately, which is a fundamental requirement for other control methods. 


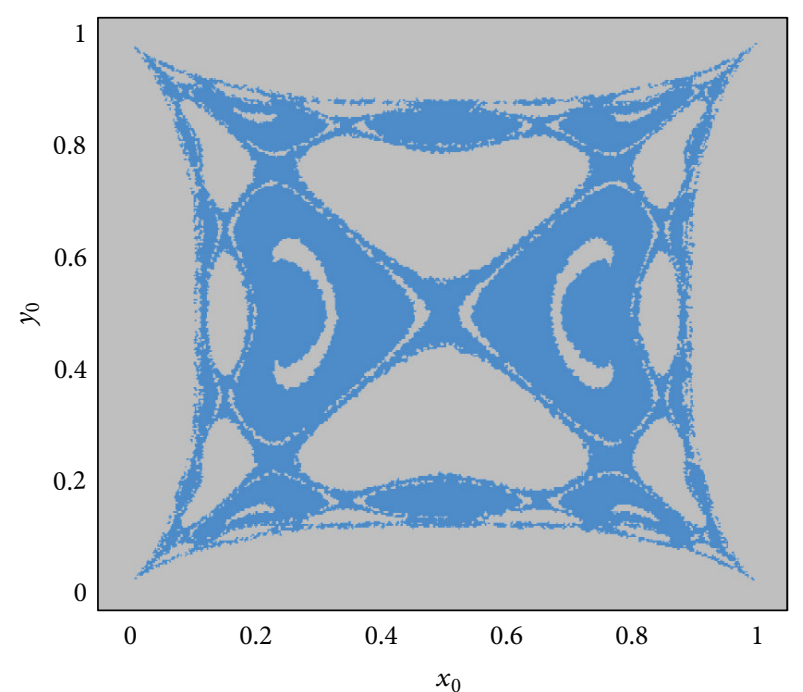

(a)

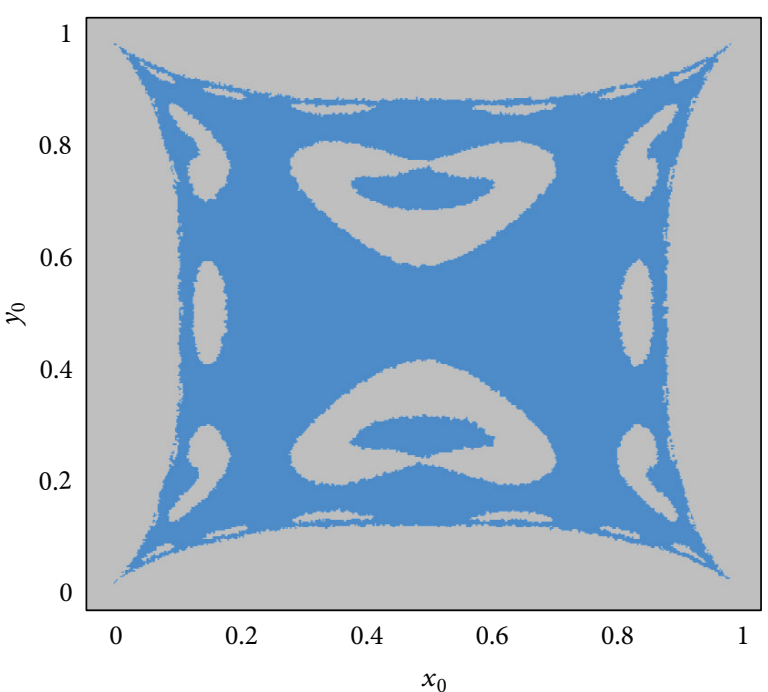

(b)

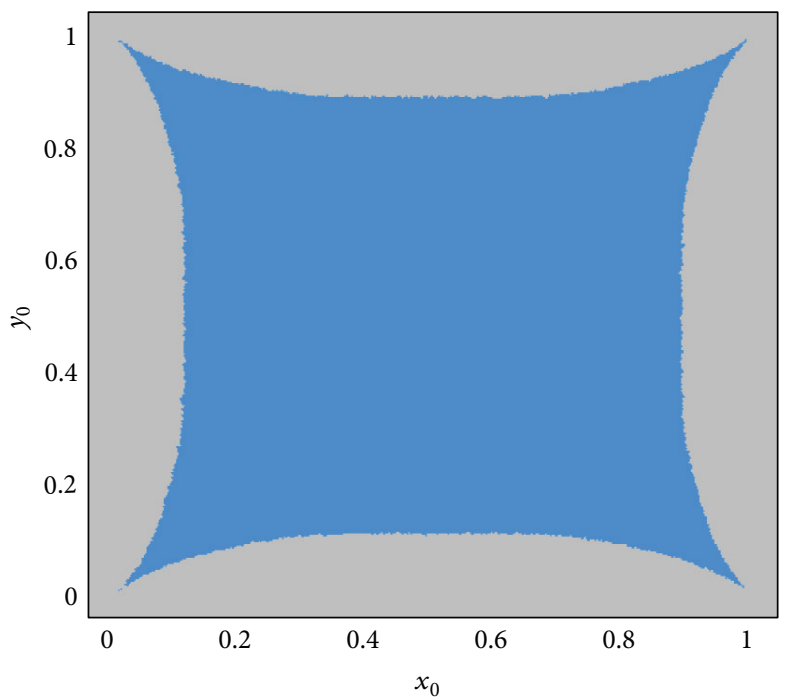

(c)

Figure 13: $\mathrm{BA}\left(P_{1}\right)$ for $E=0.25$. (b) $\mathrm{BA}\left(P_{1}\right)$ for $E=0.45$. (c) $\mathrm{BA}\left(P_{1}\right)$ for $E=0.65 . r=2.8$ and $\epsilon=1.5$ for all cases.

The applicability of the proposed tools has been illustrated in linear and nonlinear coupling logistic maps. However, the developed tools are generic and can be applied to any system with complex scenarios.

\section{Conflict of Interests}

The authors declare that there is no conflict of interests regarding the publication of this paper.

\section{Acknowledgment}

This work was partially supported by Universidad Nacional de Colombia, Manizales, Projects 28528 and 22546, Vicerrectoría de Investigación, DIMA.

\section{References}

[1] E. Ott, Chaos in Dynamical Systems, Cambridge University Press, Cambridge, UK, 2002.

[2] R. L. Devaney, An Introduction to Chaotic Dynamical Systems, Addison-Wesley, Reading, Mass, USA, 2nd edition, 1989.

[3] E. Mosekilde, Topics in Nonlinear Dynamics-Applications to Physics, Biology and Economic Systems, World Scientific, Singapore, 1996.

[4] S. Wiggins, Global Bifurcations and Chaos: Analytical Methods, Springer, New York, NY, USA, 1988.

[5] Z. T. Zhusubaliyev and E. Mosekilde, Bifurcations and Chaos in Piecewise-Smooth Dynamical Systems, World Scientific, 2003.

[6] S. Banerjee and G. Verghese, Nonlinear Phenomena in Power Electronics Attractors, Bifurcations, Chaos, and Nonlinear Control, IEEE Press, New York, NY, USA, 2001. 
[7] A. Polynikis, M. di Bernardo, and S. J. Hogan, "Synchronizability of coupled PWL maps," Chaos, Solitons and Fractals, vol. 41, no. 3, pp. 1353-1367, 2009.

[8] Z. T. Zhusubaliyev and E. Mosekilde, "Multilayered tori in a system of two coupled logistic maps," Physics Letters A, vol. 373, no. 10, pp. 946-951, 2009.

[9] Z. T. Zhusubaliyev and E. Mosekilde, "Novel routes to chaos through torus breakdown in non-invertible maps," Physica D: Nonlinear Phenomena, vol. 238, no. 5, pp. 589-602, 2009.

[10] L. M. Pecora and T. L. Carroll, "Pseudoperiodic driving: eliminating multiple domains of attraction using chaos," Physical Review Letters, vol. 67, no. 8, article 945, 1991.

[11] K. Kaneko, "Dominance of milnor attractors and noise-induced selection in a multiattractor system," Physical Review Letters, vol. 78, no. 14, pp. 2736-2739, 1997.

[12] S. Kraut, U. Feudel, and C. Grebogi, "Preference of attractors in noisy multistable systems," Physical Review E, vol. 59, no. 5, pp. 5253-5260, 1999.

[13] A. N. Pisarchik and B. K. Goswami, "Annihilation of one of the coexisting attractors in a bistable system," Physical Review Letters, vol. 84, no. 7, pp. 1423-1426, 2000.

[14] B. K. Goswami and A. N. Pisarchik, "Controlling multistability by small periodic perturbation," International Journal of Bifurcation and Chaos, vol. 18, no. 6, pp. 1645-1673, 2008.

[15] A. N. Pisarchik and R. Jaimes-Reategui, "Control of basins of attraction in a multistable fiber laser," Physics Letters A, vol. 374, no. 2, pp. 228-234, 2009.

[16] Y. Liu, M. Wiercigroch, J. Ing, and E. Pavlovskaia, "Intermittent control of coexisting attractors," Philosophical Transactions of the Royal Society A, vol. 371, no. 1993, 15 pages, 2013.

[17] R. Sevilla-Escoboza, A. N. Pisarchik, R. Jaimes-Reátegui, and G. Huerta-Cuellar, "Selective monostability in multi-stable systems," Proceedings of the Royal Society A, vol. 471, no. 2180, 2015.

[18] A. N. Pisarchik and U. Feudel, "Control of multistability," Physics Reports, vol. 540, no. 4, pp. 167-218, 2014.

[19] F. Angulo, Dynamical analisys of PWM-controlled power electronic converters based on the zero average dynamics (ZAD) strategy [Ph.D. thesis], Polytechnic University of Catalonia, Barcelona, Spain, 2004 (Spanish), http://www.tdx.cesca.es/ TDX-0727104-095928/.

[20] A. El Aroudi, F. Angulo, G. Olivar, B. G. M. Robert, and M. Feki, "Stabilizing a two-cell DC-DC buck converter by fixed point induced control," International Journal of Bifurcation and Chaos, vol. 19, no. 6, pp. 2043-2057, 2009.

[21] F. E. Hoyos, A. Rincón, J. A. Taborda, N. Toro, and F. Angulo, "Adaptive quasi-sliding mode control for permanent magnet DC motor," Mathematical Problems in Engineering, vol. 2013, Article ID 693685, 12 pages, 2013.

[22] L. Gardini, R. H. Abraham, D. Fournier Prunaret, and R. J. Record, "A double logistic map," International Journal of Bifurcation and Chaos, vol. 4, no. 1, pp. 145-176, 1994.

[23] A. N. Pisarchik, "Controlling the multistability of nonlinear systems with coexisting attractors," Physical Review E, vol. 64, no. 4, Article ID 046203, 2001.

[24] E. N. Egorov and A. A. Koronovskii, "Dynamical control in multistable systems," Technical Physics Letters, vol. 30, no. 3, pp. 186-189, 2004. 


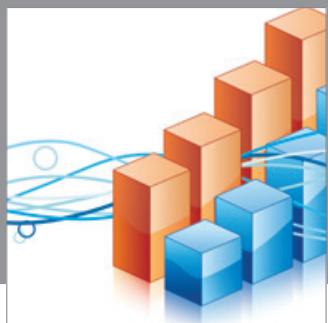

Advances in

Operations Research

mansans

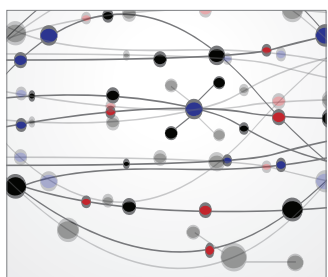

The Scientific World Journal
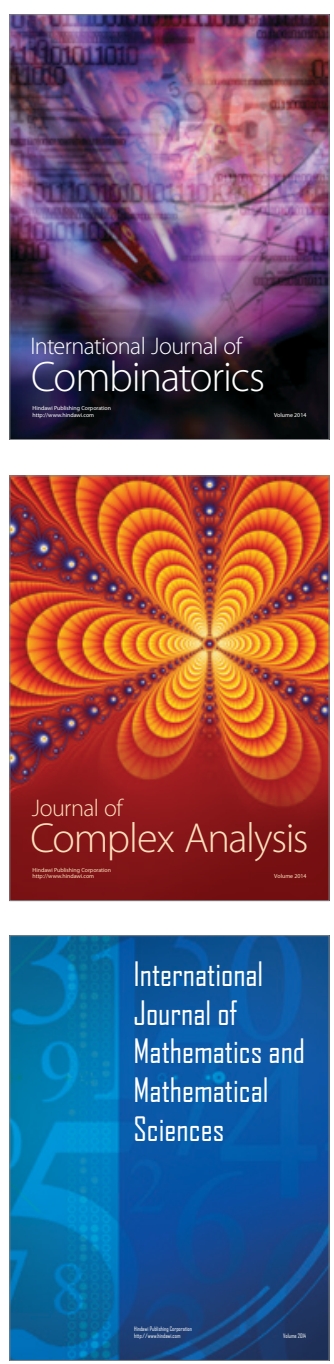
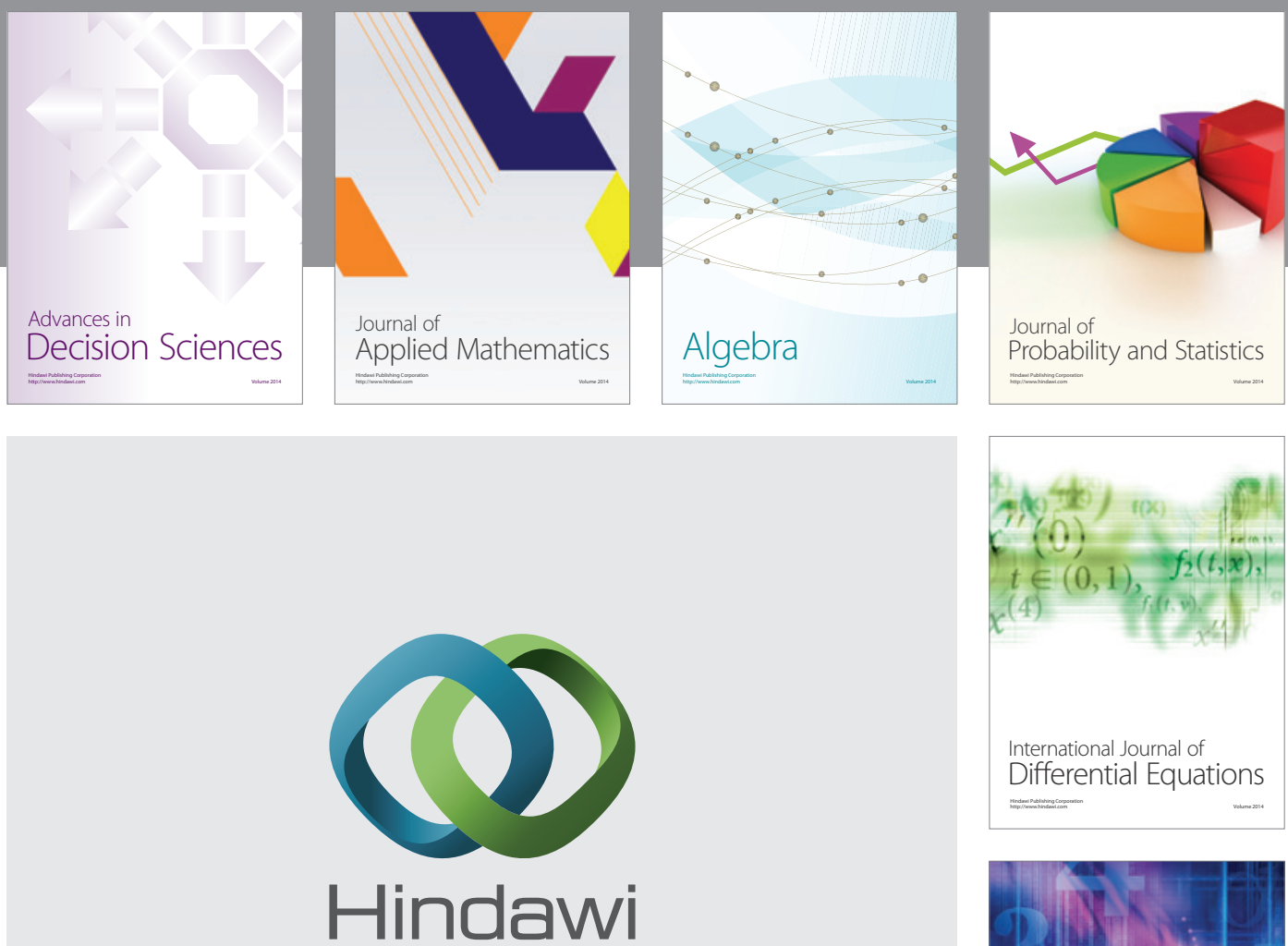

Submit your manuscripts at http://www.hindawi.com
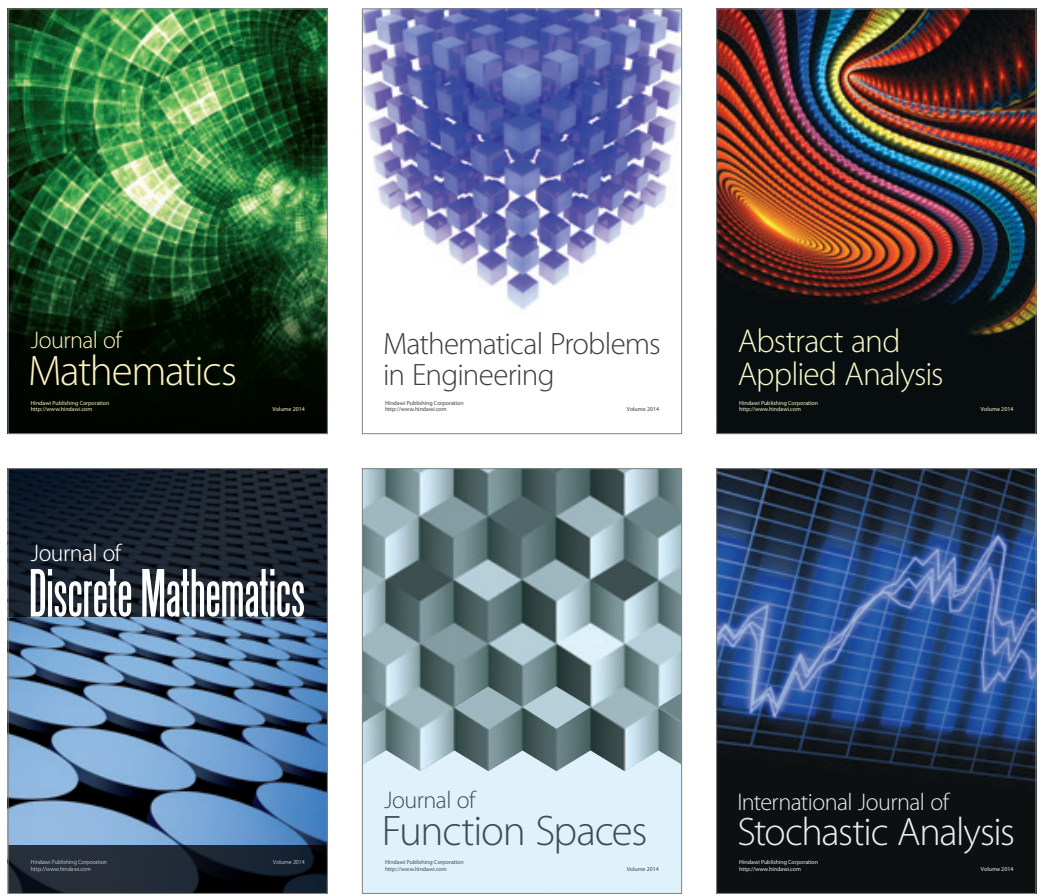

Journal of

Function Spaces

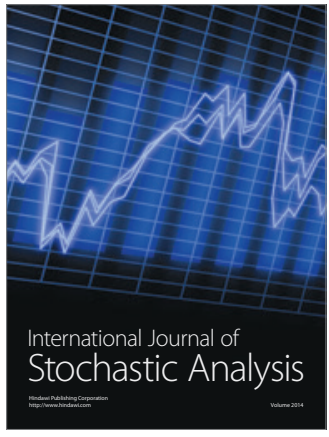

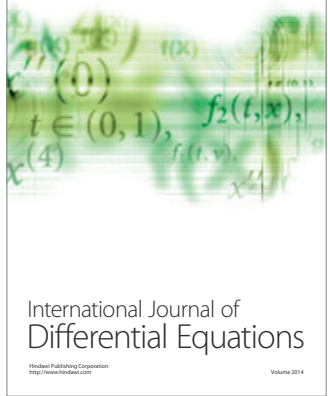
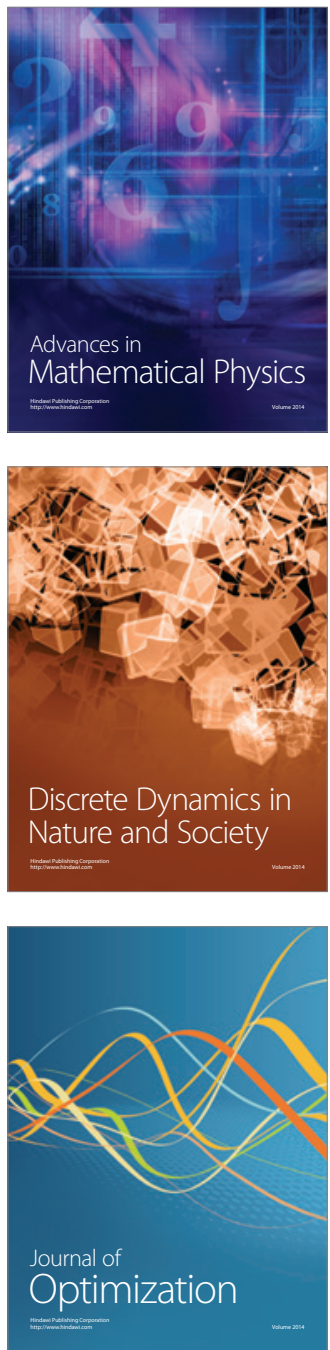